\title{
A 3-year-old female with cough, dyspnoea and low-grade fever
}

\author{
J.V. Been*, S.G. Robben* , T. Delhaas ${ }^{\mp}$, P. Theunissen+, E. Dompeling $^{\S}$ and Q. Jöbsis ${ }^{\S}$
}

\section{CASE REPORT}

A 3-yr-old female presented at the emergency dept with a 24-h history of dyspnoea, productive cough, bilious vomiting and a low-grade fever. At physical examination, nasal flaring and mild chest retractions were noted. No stridor or wheezing was present and oxygen saturation was normal. Heart rate was $120 \mathrm{bpm}$ in the presence of a low-grade fever $\left(38.6^{\circ} \mathrm{C}\right)$. Decreased breath sounds were heard over the right hemithorax, while no asymmetry was noted on either chest inspection or percussion. Otherwise, the findings of physical examination were normal.

Laboratory investigation showed no signs of infection. A chest radiograph was performed (fig. 1). As a result of the dyspnoea, the patient was admitted and azithromycin was commenced to cover possible Mycoplasma pneumoniae. However, serological screening for Mycoplasma antibodies was negative. Furthermore, no pathogen was cultured from either blood or pharynx.

During admission an additional history was taken, in which the mother reported that the child had previously choked upon eating a piece of candy, 1 week before the onset of symptoms. To rule out the presence of a foreign body, which could explain both the symptoms and the abnormalities seen on chest

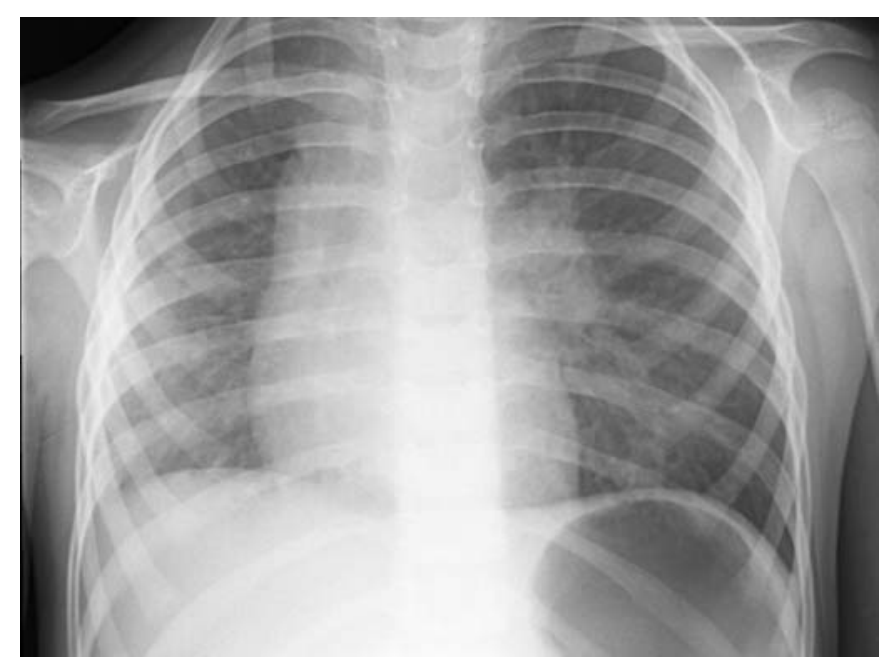

FIGURE 1. Chest radiograph at presentation. radiography, the patient was transferred to a tertiary centre for bronchoscopic evaluation. At bronchoscopy, purulent sputum was noted in the left main bronchus but no foreign body was found. Laboratory investigation was repeated, showing C-reactive protein (CRP) of $16 \mathrm{mg} \cdot \mathrm{L}^{-1}$ without any other abnormalities. Moraxella catarrhalis was cultured from the sputum obtained at bronchoscopy. This pathogen was treated with amoxicillin and clavulanic acid for 7 days, although no clear signs of infection were demonstrated. As no explanation for the clinical situation was found, computed tomography (CT) of the chest was performed 5 days after admission (fig. 2).

Additional review of the medical history revealed that the patient had been previously admitted during the neonatal period. She was small for gestational age and experienced hypoglycaemias for which she was treated with glucose infusion. Approximately $24 \mathrm{~h}$ after birth, the patient experienced a cyanotic incident with irregular breathing movements and hypotonia. Oxygen saturation was $40 \%$ and increased slowly upon administration of oxygen via a face mask.

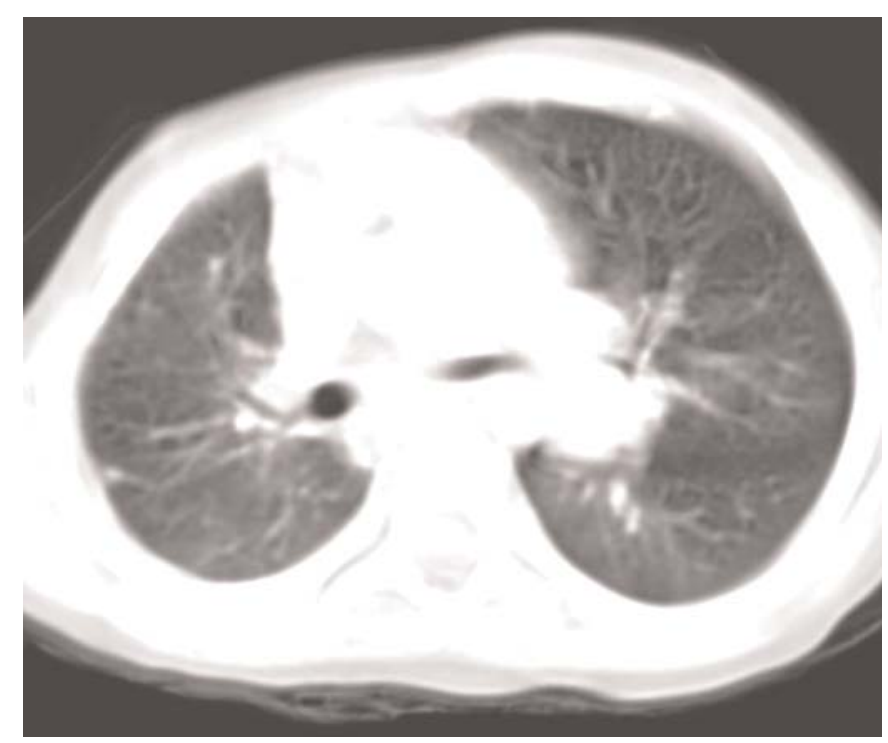

FIGURE 2. Non-contrast-enhanced computed tomography scan of the chest in a pulmonary window setting.

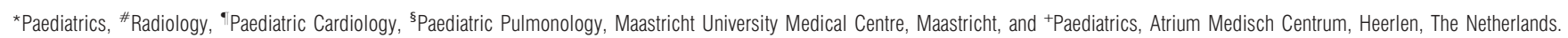
STATEMENT OF INTEREST: None declared.

CORRESPONDENCE: J.V. Been, Dept of Paediatrics, Maastricht University Medical Centre, P0 Box 5800, 6202 AZ Maastricht, The Netherlands. Fax: 31 433875246. E-mail: jasper.been@mumc.nl
} 


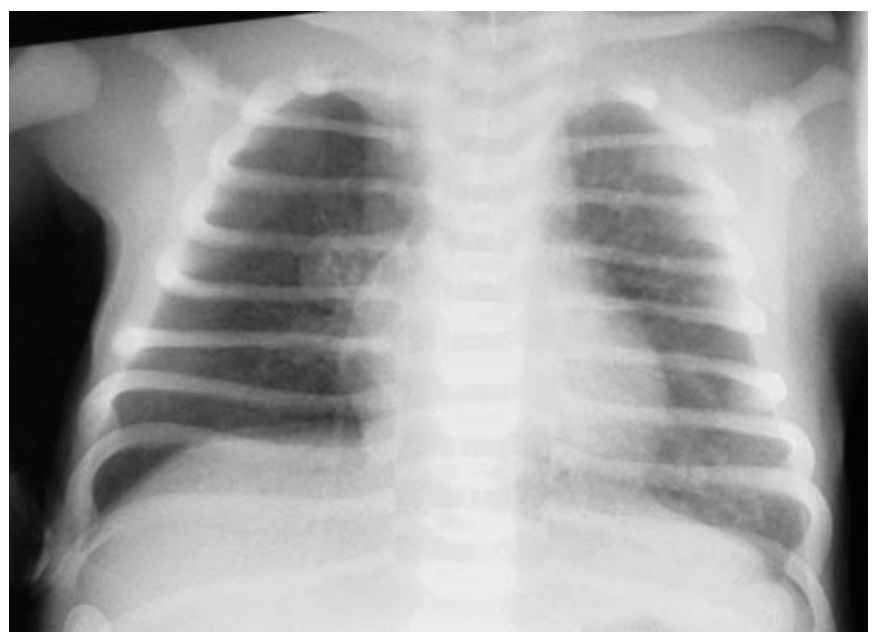

Laboratory investigation was performed at that time, showing CRP of $11 \mathrm{mg} \cdot \mathrm{L}^{-1}$ and leukocytosis of $24.7 \times 10^{9}$ cells $\cdot \mathrm{L}^{-1}$ with normal differentiation. Blood gas analysis showed a $\mathrm{pH}$ of 7.37, a carbon dioxide tension of $5.1 \mathrm{kPa}$ and a bicarbonate level of $21 \mathrm{mmol} \cdot \mathrm{L}^{-1}$. A chest radiograph was also performed (fig. 3). Over the next few days, the patient experienced several similar episodes, yet these diminished and eventually subsided over time. After a hospital admission of 3 weeks she was discharged in good clinical condition, yet without a diagnosis having been made.

FIGURE 3. Chest radiograph taken on the first day of life in the neonatal ward

\section{BEFORE TURNING THE PAGE, INTERPRET THE CHEST RADIOGRAPHS AND CT (FIGS 1-3), AND SUGGEST A DIAGNOSIS.}




\section{INTERPRETATION}

The chest radiograph at presentation (fig. 1) showed displacement of the heart and mediastinum to the right. The left lung appeared somewhat hyperlucent with increased intercostal distances present. There was also some retrosternal herniation of the left lung to the right. These radiological signs were suggestive of either air trapping of the left lung or atelectasis of the right lung. However, fluoroscopy showed symmetric diaphragmatic excursions without any signs of air trapping. The left hilum was enlarged, whereas the right hilum could not be analysed due to overprojection of the heart. A foreign body or mucus can cause both air trapping and atelectasis. Therefore, this radiograph justified bronchoscopy, although the enlarged left hilum and the symmetric diaphragmatic excursions were still not explained. Swyer-James (MacLeod) syndrome seemed less likely because the classical signs (small hyperlucent lung and air trapping) were not present.

The chest radiograph taken on the first day of life (fig. 3) was initially reported as normal. However, the right lung was more lucent than the left lung due to an overall decrease in pulmonary vasculature (oligaemia) on the right with a normal lung volume. Obviously, the mediastinal shift and decreased volume of the right hemithorax as seen in figure 1 had not yet developed. These findings favoured a congenital aetiology. Swyer-James (MacLeod) syndrome became more unlikely because this is an acquired condition in which the lungs are assumed to be normal at birth.

The native CT scan of the chest (fig. 2) showed a normal bronchial tree without bronchiectasis, focal pulmonary abnormalities or pleural effusion. The volume of the right hemithorax was smaller than the left hemithorax. The left hilum was enlarged, as in the chest radiograph. Mediastinal structures could not be evaluated in this window setting without intravenous contrast medium.

Figure 4 shows a contrast-enhanced CT scan of the same patient, made during the same session as figure 2 . The left pulmonary artery is enlarged, whereas the right pulmonary artery is absent, pointing to the diagnosis of unilateral absence of a pulmonary artery (UAPA). Cardiac ultrasonography

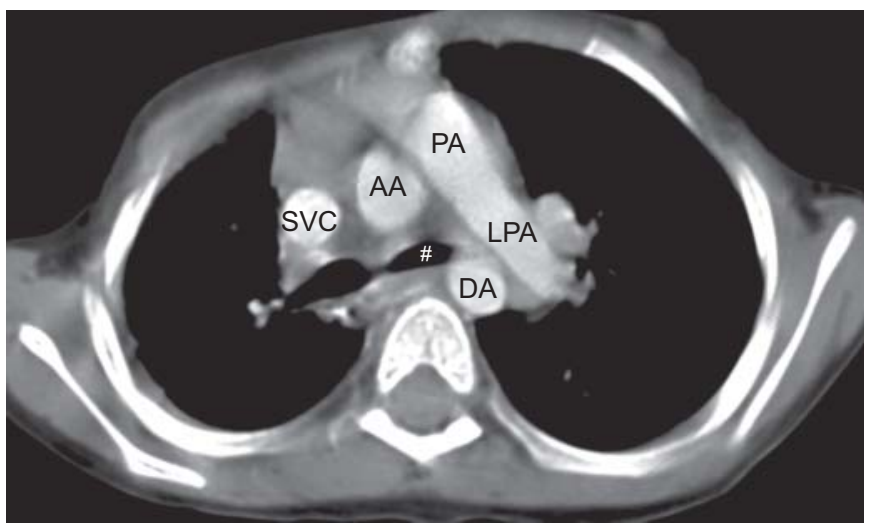

FIGURE 4. Computed tomography of the chest with intravenous contrast in a mediastinal window setting. ${ }^{*}$ : the normal anatomic location of the right pulmonary artery; it is absent here. SVC: superior vena cava; AA: ascending aorta; DA: descending aorta; PA: pulmonary artery; LPA: left pulmonary artery. showed no associated congenital heart defect, while subsequent angiography confirmed the diagnosis of absent right pulmonary artery, absent major aortopulmonary collateral arteries, and the remains of a right-sided ductus arteriosus on the brachiocephalic truncus (fig. 5). There was no pulmonary hypertension. No associated abnormalities were found on additional ultrasonography of the abdominal vasculature and kidneys.

\section{Diagnosis: Unilateral (right-sided) absence of the pulmon- ary artery.}

\section{CLINICAL COURSE}

After a hospital stay of 10 days, the patient was discharged in a good clinical condition. Over the following year, she experienced recurrent upper airway infections. As there were no symptoms at 2 yrs of follow-up, it was decided that no interventions should be performed. The subject is now $8 \mathrm{yrs}$ of age; no symptoms attributable to UAPA are present and no abnormalities were noted at follow-up.

\section{DISCUSSION}

UAPA is a rare anomaly, with an estimated prevalence of one in 150,000 [1]. In the majority of cases, pulmonary artery agenesis is associated with other congenital heart diseases (CHD), such as tetralogy of Fallot and septal defects. In a recent systematic literature review, 108 reported cases of UAPA without associated CHD were found [2]. In this group, the distal pulmonary artery is of ductal origin and involution of the sixth aortic arch during embryogenesis results in absence of the proximal pulmonary artery. Therefore, it has been suggested that the term UAPA should be replaced by ductal origin of the distal pulmonary artery [3]. However, if not timely diagnosed, closure of the ductus arteriosus will result in involution and eventually absence of the distal pulmonary artery. Hence, the present authors feel that the term UAPA is justified for these patients.

UAPA may present with recurrent pulmonary infections $[2,4-$ 7], exercise intolerance or dyspnoea [2], haemoptysis [2, 6],

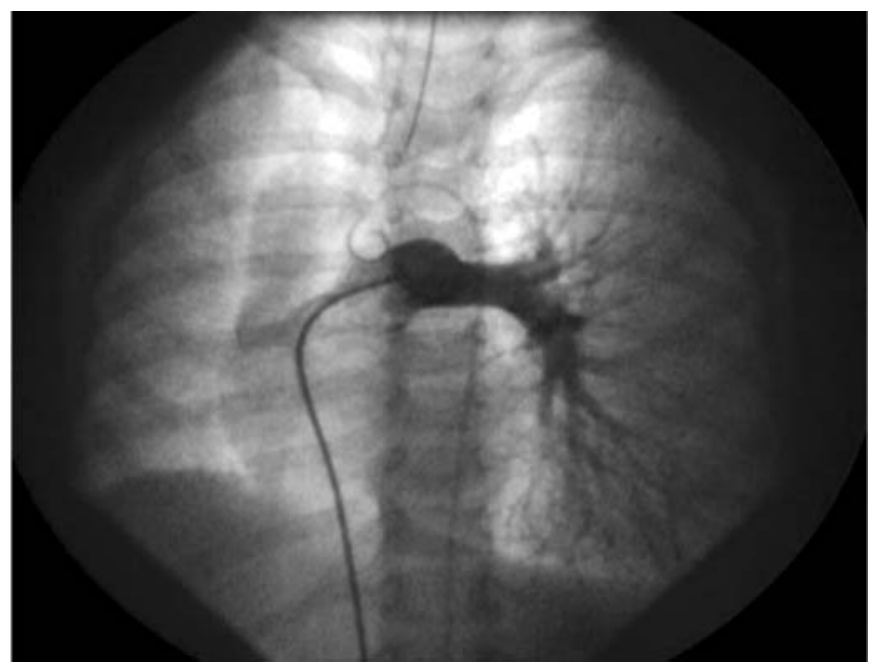

FIGURE 5. Angiography of the pulmonary artery. No opacification of the right pulmonary artery is seen. During the same procedure, an angiography of the aorta was performed without any signs of aortopulmonary collaterals. 
high-altitude pulmonary oedema [2], or pulmonary hypertension. Due to the unspecific symptoms associated with UAPA, the diagnosis is often delayed. Symptomatic periods prior to diagnosis of up to 39 yrs have been described [8], with the median age at diagnosis being 14 yrs [2].

Once suspected, further evaluation should be directed towards confirming the diagnosis, excluding associated abnormalities and screening for possible complications (discussed further later). In addition to chest radiography, this evaluation should include CT or magnetic resonance image scanning, echocardiography and cardiac catheterisation including pulmonary artery pressure measurement.

The history of the present patient, in addition to findings on physical examination and chest radiography, was suggestive of foreign body aspiration. A recent study showed that the combination of focal hyperinflation on chest radiography and a witnessed choking crisis has a positive predictive value of $96 \%$ of finding a foreign body on bronchoscopy [9]. Therefore, the absence of a foreign body in the present case warranted further evaluation of the unexplained signs and symptoms, eventually leading to the diagnosis.

It is interesting to note that the affected lung had a normal volume at birth, whereas the neonatal hyperlucency could be explained by the accompanying oligaemia. The lack of pulmonary vascularisation of the lung in the present patient with UAPA seemed to cause a postnatal arrest of progressive growth and alveolarisation of the lung, resulting in pulmonary hypoplasia at 3 yrs of age. At least, no other causes of acquired hypoplasia, such as severe infections, were documented.

Whether the symptoms with which the patient presented in the neonatal period may have been attributable to UAPA remains uncertain. However, the clinical history of this case underlines the importance of both early consideration of the diagnosis of UAPA and further evaluation in case of paradoxical clinical findings.

The implications of the diagnosis are important, as serious and potentially lethal complications are common. Pulmonary hypertension is found in $44 \%$ of reported UAPA patients screened for pulmonary hypertension [2]. Other possible complications include bronchiectasis, recurrent haemoptysis and cardiac failure. At the time of their report in the literature, $14 \%$ of UAPA patients had developed complications sufficiently severe to warrant surgical intervention, such as unilateral pneumectomy, lobectomy or surgical pulmonary artery revascularisation [2]. Follow-up should be directed towards early detection of complications, most importantly pulmonary hypertension. Recently, WELCH et al. [10] reported primary surgical treatment in two cases of right-sided UAPA with an uncomplicated follow-up of $2.5 \mathrm{yrs}$. Both cases were diagnosed and treated in early infancy. Treatment consisted of bridging the main pulmonary artery and the hilar right pulmonary artery using a filleted ligamentum arteriosis and a pulmonary arterial homograft patch. Increased experience with this technique and longer follow-up are needed in order to establish whether primary surgery improves the outcome of UAPA patients.

In conclusion, unilateral absence of a pulmonary artery is a rare anomaly associated with an important risk of serious complications. Diagnosis is often delayed, although in many cases, both clinical symptoms and findings on chest radiography may have been suggestive of unilateral absence of a pulmonary artery at an earlier stage. Consideration of unilateral absence of a pulmonary artery in the differential diagnosis of recurrent respiratory infections, haemoptysis, pulmonary hypertension and asymmetrical chest radiograph findings consistent with unilateral underdevelopment of the lung is important, as complications are common yet detectable and treatable at an early stage.

\section{REFERENCES}

1 Bouros D, Pare P, Panagou P, Tsintiris K, Siafakas N. The varied manifestation of pulmonary artery agenesis in adulthood. Chest 1995; 108: 670-676.

2 Ten Harkel AD, Blom NA, Ottenkamp J. Isolated unilateral absence of a pulmonary artery: a case report and review of the literature. Chest 2002; 122: 1471-1477.

3 Trivedi KR, Karamlou T, Yoo SJ, Williams WG, Freedom RM, McCrindle BW. Outcomes in 45 children with ductal origin of the distal pulmonary artery. Ann Thorac Surg 2006; 81: 950-957.

4 Stiller RJ, Soberman S, Turetsky A, Lockwood C, Haddad R. Agenesis of the pulmonary artery: an unusual cause of dyspnea in pregnancy. Am J Obstet Gynecol 1988; 158: 172-173.

5 Ferrari M, Karrazi R, Lampronti G, et al. Effect of changing position on arterial oxygenation in a patient with agenesia of the left pulmonary artery. Respiration 1997; 64: 371-374.

6 Ko T, Gatz MG, Reisz GR. Congenital unilateral absence of a pulmonary artery: a report of two adult cases. Am Rev Respir Dis 1990; 141: 795-798.

7 Shakibi JG, Rastan H, Nazarian I, Paydar M, Aryanpour I, Siassi B. Isolated unilateral absence of the pulmonary artery. Review of the world literature and guidelines for surgical repair. Jpn Heart J 1978; 19: 439-451.

8 Scheuch RW, Simon-Gabor M, Weinberg HR, Eisenberg H. Left pulmonary artery agenesis. N Y State J Med 1988; 88: 200-201.

9 Heyer CM, Bollmeier ME, Rossler L, et al. Evaluation of clinical, radiologic, and laboratory prebronchoscopy findings in children with suspected foreign body aspiration. J Pediatr Surg 2006; 41: 1882-1888.

10 Welch K, Hanley F, Johnston T, Cailes C, Shah MJ. Isolated unilateral absence of right proximal pulmonary artery: surgical repair and follow-up. Ann Thorac Surg 2005; 79: 1399-1402. 